\title{
Causal Effects of Sleep Traits on Ischemic Stroke and Its Subtypes: A Mendelian Randomization Study
}

This article was published in the following Dove Press journal: Nature and Science of Sleep

\begin{abstract}
Huan Cai ${ }^{1, *}$
Jialin Liang ${ }^{2, *}$

Zhonghua Liu'

Liang Fang'

Jinghong Zheng ${ }^{3}$

Jiahe $\mathrm{Xu}^{3}$

Liyi Chen'

Wen Sun ${ }^{4}$

Hao Zhang $\mathbb{D}^{5}$

'Department of Rehabilitation, Zhongshan City People's Hospital, Zhongshan 528403,

Guangdong, People's Republic of China;

${ }^{2}$ Department of Endocrinology and

Metabolism, Zhongshan City People's

Hospital, Zhongshan 528403, Guangdong,

People's Republic of China; ${ }^{3}$ Department of

Neurology, Zhongshan City People's

Hospital, Zhongshan 528403, Guangdong,

People's Republic of China; ${ }^{4}$ Division of Life

Sciences and Medicine, Stroke Center and

Department of Neurology, The First

Affiliated Hospital of USTC, University of

Science and Technology of China, Hefei

230026, Anhui, People's Republic of China;

${ }^{5}$ Department of Neurology, Affiliated

Hangzhou First People's Hospital, Zhejiang

University School of Medicine, Hangzhou

310006, Zhejiang, People's Republic of China

*These authors contributed equally to this work
\end{abstract}

Correspondence: Hao Zhang Department of Neurology, Affiliated

Hangzhou First People's Hospital,

Zhejiang University School of Medicine,

Hangzhou 310006, Zhejiang, People's

Republic of China

Tel +86-13450989238

Fax +86 760-87727692

Email zh_hao_neurol@I63.com

Zhonghua Liu

Department of Rehabilitation, Zhongshan

City People's Hospital, Zhongshan 528403,

Guangdong, People's Republic of China

Tel 86-I 3923308898

Email zhonghua_reha@I63.com
Background: Unfavorable sleep habits have been linked with ischemic stroke in observational studies, but the causality remains unclear. The aim of this study is to investigate the potential causal role of three sleep traits, including sleep duration, insomnia, and chronotype, in ischemic stroke and its subtypes.

Methods: We conducted two-sample Mendelian randomization (MR) analysis using single nucleotide polymorphisms associated with sleep duration, insomnia, and chronotype as instruments to estimate causal associations with ischemic stroke and its subtypes, among 34,217 ischemic stroke cases and 406,111 controls from the MEGASTROKE consortium. Inverse-variance weighted method was used as the main analyses. Alternative MR methods and sensitivity analyses were further performed.

Results: We found suggestive evidence that per doubling of genetic liability for short sleep duration (odds ratio [OR], 1.27; 95\% confidence interval [CI], 1.01-1.58) and frequent insomnia symptoms (OR, 1.19; 95\% CI, 1.00-1.41) were associated with a modest increase in risk of large artery stroke (LAS) but not with small vessel stroke, cardioembolic stroke, or any ischemic stroke. The association of frequent insomnia symptoms with LAS was stronger after the exclusion of the outlier (OR, $1.25 ; 95 \% \mathrm{CI}$, 1.04-1.50). No significant association was observed for chronotype with any ischemic stroke subtype. Results were overall robust to sensitivity analyses, and there was little evidence of horizontal pleiotropy.

Conclusion: We provided suggestive evidence for a potential causal role of short sleep duration and insomnia symptoms in LAS. Future researches are required to investigate whether improved sleep habits could help to mitigate LAS risk.

Keywords: sleep, large artery stroke, ischemic stroke, Mendelian randomization

\section{Introduction}

Unfavorable sleep habits are important public health problems worldwide. In humans, much of the literature on the relationship between sleep and stroke has focused primarily on obstructive sleep apnoea. ${ }^{1}$ However, the impact of non-apnoea related sleep traits, such as sleep duration, insomnia, and chronotype (morning or evening preference), in ischemic stroke remains unclear. ${ }^{1}$ In recent years, there are numerous reported associations between unfavorable sleep habits and ischemic stroke, but the results are conflicting ${ }^{2-10}$ and might be subjected to limitations in observational studies of confounding and reverse causation. Moreover, ischemic stroke is a heterogeneous disease. Different ischemic stroke subtypes have distinct pathogenesis and pathophysiology that likely reflect differences in the importance of underlying risk factors, as well as in genetic determinants. ${ }^{11-13}$ However, less 
investigation has been done into the potential adverse effects of unfavorable sleep habits on stroke subtypes.

Mendelian randomization (MR) utilizes genetic variants associated with modifiable exposure (eg, sleep traits) as instruments to infer causality. Because of the random assortment of genetic variants at conception, the MR design is less susceptible to confounding and reverse causation. ${ }^{14}$ Recently, large-scale genome-wide association studies (GWAS) have identified genetic variants robustly associated with sleep duration, insomnia, and chronotype. $^{15-17}$ Here, we applied two-sample MR to comprehensively investigate the potential causal role of three sleep traits, including sleep duration, insomnia, and chronotype, in ischemic stroke and its subtypes.

\section{Materials and Methods}

\section{Data Source and Single Nucleotide Polymorphisms Selection}

Summary-level data for genetic associations with sleep traits and ischemic stroke and its subtypes have been made publicly available online (Supplementary Table 1). Participants in our MR analyses were predominantly of European ancestry. Summary statistics data for the associations of each single nucleotide polymorphism (SNP) with ischemic stroke and its subtypes were derived from the GWAS meta-analysis by the MEGASTROKE consortium, including up to 34,217 ischemic stroke cases and 406,111 stroke-free controls of European ancestry. Participants were subdivided into large artery stroke (LAS) ( $n=4373$ cases/146,392 controls), small vessel stroke (SVS) ( $\mathrm{n}=5386$ cases/192,662 controls), and cardioembolic stroke (CES) ( $n=7193$ cases/204,570 controls) based on the Trial of Org 10,172 in Acute Stroke Treatment criteria. ${ }^{11,18}$

The previously published SNPs associated with each of the sleep phenotype were selected as the instruments. Sleep duration was assessed by asking: "About how many hours sleep do you get in every $24 \mathrm{~h}$ ? (please include naps)" in the UK Biobank. ${ }^{15}$ Effect size estimates for SNPs associated with continuous sleep duration (number of hours sleep; $n=446,118$ ), short sleep duration (6h or less; $\mathrm{n}=106,192$ cases/305,742 controls), and long sleep duration ( $9 \mathrm{~h}$ or more; $\mathrm{n}=34,184$ cases/305,742 controls) at a genome-wide threshold of significance $\left(p<5 \times 10^{-8}\right)$ were available. $^{15}$

Insomnia symptoms were assessed by asking: "Do you have trouble falling asleep at night, or do you wake up in the middle of the night?" with responses of "never/rarely," "sometimes," "usually," and "prefer not to answer." Those responded "prefer not to answer" were set to missing. ${ }^{16}$ Insomnia symptoms associated SNPs were identified in the UK Biobank and were confirmed in the HUNT, ${ }^{19}$ and Partners Biobank studies from two parallel GWAS: (1) frequent insomnia symptoms ("never/rarely" versus "usually"; $n=146,410$ cases/108,357 controls) and (2) any insomnia symptoms ("never/rarely" versus"sometimes"/“usually"; $\mathrm{n}=362,171$ cases/170,207 controls). ${ }^{16}$

Genetic association estimates for chronotype were taken from a GWAS meta-analysis performed in the UK Biobank and 23andMe $(\mathrm{n}=372,765$ cases $/ 278,530$ controls). ${ }^{17}$ In the 23 andMe cohort, chronotype (evening or morning preference) was assessed by asking: "Are you naturally a night person or a morning person?" In the UK Biobank, participants were asked: "Do you consider yourself to be?" with one of the six possible answer: "Definitely a 'morning' person," "More a 'morning' than 'evening' person," "More an 'evening' than a 'morning' person," "Definitely an 'evening' person," "Do not know" or "Prefer not to answer". Those answering "Definitely an 'evening' person" and "More an 'evening' than a 'morning' person" were defined as evening persons (controls) and those answering "Definitely a 'morning' person" and "More a 'morning' than 'evening' person" were defined as morning persons (cases).

For each trait, we restricted instruments to independent SNPs not in linkage disequilibrium $\left(r^{2}<0.1\right)$. Where SNPs for the sleep phenotype were unavailable in the outcome GWAS summary statistics, we deleted them. Thus, 27 SNPs were used to instrument short sleep duration (Supplementary Table 2), 6 SNPs to instrument long sleep duration, 75 SNPs to continuous sleep duration, 56 SNPs to insomnia symptoms (Supplementary Table 3), and 332 SNPs to chronotype. Ethical approval and informed consent have been obtained in the original studies.

\section{Statistical Analyses}

We used inverse-variance weighted (IVW) method as the main analyses to obtain estimates for the causal effect of sleep traits on ischemic stroke risk. This method will return an accurate estimate in the absence of horizontal pleiotropy, or when horizontal pleiotropy is balanced. ${ }^{14}$ We therefore compared IVW results with several robust MR methods for sensitivity tests. These methods include: (1) weighted median estimate (WME), which requires that at least half of the instrument variables are valid; ${ }^{20}$ (2) 
MR-Egger method, which allows for directional pleiotropic effects; ${ }^{21}$ (3) MR-Robust Adjusted Profile Score (MRRAPS), which provides unbiased estimates in the presence of many weak instruments. ${ }^{22}$ We also applied MRPleiotropy Residual Sum and Outlier (MR-PRESSO) approach to identify and correct for potential outliers. ${ }^{23}$

Heterogeneity of individual genetic variants was estimated by Cochran's $Q$ statistic. In order to ensure no evidence of directional pleiotropy for the associations of sleep traits with ischemic stroke and its subtypes, MREgger intercept tests of deviation from the null were conducted. For further interpretation, we also produced scatterplots, forest plots, funnel plots and leave-one-out plots. Finally, Steiger tests were utilized to verify the causal direction between the exposures and outcomes. ${ }^{24}$ All results are presented per hour increment in continuous sleep duration and per doubling in the odds of dichotomous exposures (ie, short sleep duration, long sleep duration, insomnia symptoms, and chronotype) as recommended elsewhere. ${ }^{25}$ In other words, causal effect estimates (ie, odds ratio [OR] for LAS) were rescaled to be interpreted for each doubling of genetic liability for dichotomous exposures. All statistical analyses were conducted using the TwoSampleMR ${ }^{26}$ and MR-PRESSO ${ }^{23}$ packages in $\mathrm{R}$ version 3.5.3.

\section{Results \\ Sleep Duration}

As reflected in Figure 1, we found evidence of a suggestive adverse causal effect of short sleep duration on LAS in the main analysis (IVW OR, 1.27 for LAS per doubling of genetic liability for short sleep duration; 95\% confidence interval [CI], 1.01-1.58; $p=0.038$; Supplementary Figure 1). The association of short sleep duration with LAS was consistent in complementary analysis using the MR-RAPS method (OR, 1.31; 95\% CI, 1.03-1.66; $p=0.026$ ). Results from WME and MR-Egger analysis yielded a similar pattern of effects, although with broader CIs due to the lower statistical power. The forest plot, funnel plot, and leave-one-out plot did not reveal individual SNP driving the overall association of short sleep duration with LAS (Supplementary Figure 2 through 4). However, the main MR analyses and sensitivity analyses showed no significant association of genetically determined short sleep duration with SVS, CES, and any ischemic stroke (Figure 1). Accordingly, no obvious heterogeneity was observed for the short sleep duration associated with SNPs (Table 1). MR-Egger intercept tests were consistent with the null, indicating no evidence of directional pleiotropy (all $p$ for intercept $\geq 0.480$; Table 1). Furthermore, Steiger tests showed no evidence that ischemic stroke and its subtypes contributed to short sleep duration (Supplementary Table 4).

Little evidence for causal effects of long sleep duration and continuous sleep on any ischemic stroke subtype was observed (all $p>0.05$; Supplementary Table 5). Similarly, the Cochran's $Q$ statistic and the MR-Egger intercept indicated no notable heterogeneity and directional pleiotropy (Supplementary Tables 6 and 7).

\section{Insomnia Symptoms}

Findings of the association of short sleep duration with LAS risk were supported using genetic variants associated with frequent insomnia symptoms, with some evidence of a possible adverse effect of frequent insomnia symptoms on LAS (IVW OR, 1.19; 95\% CI, 1.00-1.41, per doubling of genetic liability for frequent insomnia symptoms; $p=$ 0.047; Figure 2). The association was still existed and even stronger after the exclusion of the outlier in NAB2 (rs324017) detected by the MR-PRESSO (OR, 1.25; 95\% CI, 1.04-1.50; $p=0.023$; Supplementary Figure 5). Results for frequent insomnia symptoms and LAS were broadly consistent in sensitivity analyses using the alternative MR methods, although with less-precise estimates. Likewise, the association persisted when using the SNPs associated with any insomnia symptoms (Supplementary Table 5). Conversely, there was no clear evidence to suggest a causal effect of insomnia symptoms on SVS, CES, and any ischemic stroke.

MR-Egger intercept tests suggested no horizontal pleiotropy (all $p$ for intercept $\geq 0.445$ ). For frequent insomnia symptoms, there was moderate heterogeneity for LAS $(Q=76.02, p=0.032$; Table 1$)$, but the heterogeneity was eliminated when the outlier SNP rs324017 was pruned $(Q=61.11, p=0.236$; Supplementary Figure 6 through 8). Furthermore, Steiger tests showed no evidence that ischemic stroke and its subtypes contributed to insomnia symptoms (Supplementary Table 4).

\section{Chronotype}

A genetically predicted per doubling of genetic liability for morning preference was not associated with any ischemic stroke subtype (Supplementary Table 5). The lack of association remained in sensitivity analyses. There was no evidence of directional pleiotropy from any of the 
Outcome

Large Artery Stroke

IVW

MR-RAPS

WME

MR-Egger

Small Vessel Stroke

IVW

MR-RAPS

WME

MR-Egger

Cardioembolic Stroke

IVW

MR-RAPS

WME

MR-Egger

Any Ischemic Stroke IVW

MR-RAPS

WME

MR-Egger
SNPs

OR $(95 \% \mathrm{Cl})$

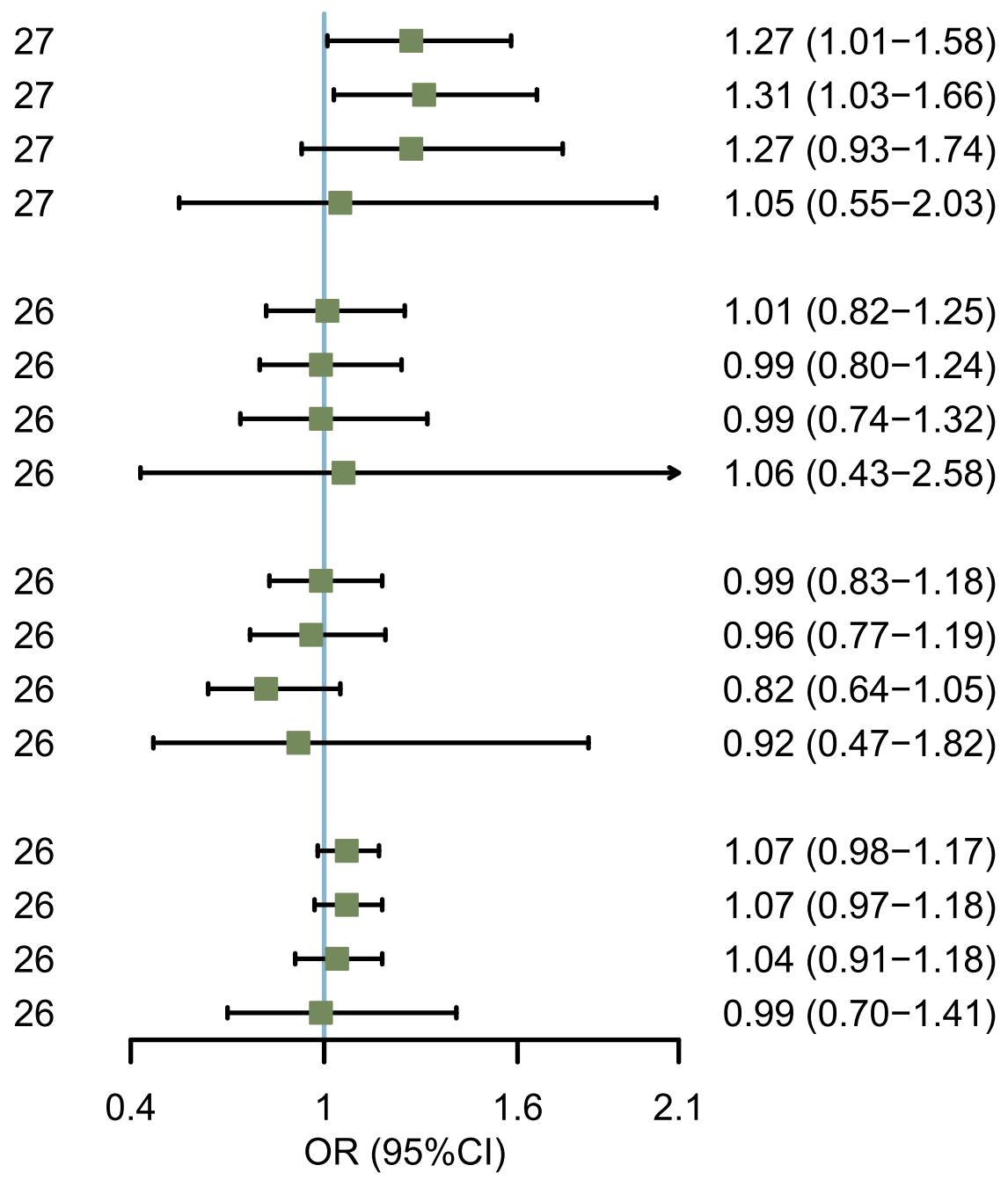

Figure I MR estimates of short sleep duration with ischemic stroke and its subtypes from the main analysis (IVW) and sensitivity analysis (MR.RAPS, WME, and MR-Egger). Data is displayed as $\mathrm{OR}$ and $95 \% \mathrm{Cl}$ per doubling of genetic liability for short sleep duration.

Abbreviations: $\mathrm{Cl}$, confidence interval; IVW, inverse-variance weighted method; MR, Mendelian randomization; MR.RAPS, MR-robust adjusted profile score; OR, odds ratio; SNP, single nucleotide polymorphism; WME, weighted median estimate.

MR-Egger intercept (Supplementary Table 7), but substantial heterogeneity was observed (Supplementary Table 6).

\section{Discussion}

We applied two-sample MR approach to make inference about the potential causal effects of three sleep phenotypes on ischemic stroke and subtypes risk. Our MR analyses demonstrated that genetic liability to short sleep duration and insomnia symptoms were potential, yet to be confirmed, causally associated with an increased risk of LAS, but not SVS, CES, or any ischemic stroke. These results were overall robust to sensitivity analyses. However, no significant causal link was observed between chronotype (morning preference) and any ischemic stroke subtype. Collectively, our study highlights sleep as a potentially modifiable risk factor of LAS.

Previous observational studies reporting the relevance of sleep and ischemic stroke have yielded inconsistent results. Short sleep duration was found to be an independent risk factor of stroke events in hypertensive patients. ${ }^{2}$ Another cohort of 11,367 Japanese population also showed a higher risk of stroke in men who sleep less than 6 hours a day. ${ }^{4}$ Similarly, in a multiethnic sample of US adults, insufficient rest or sleep was independently related to stroke. $^{27}$ However, race disparity existed in the REGARDS study, which reported short sleep duration among black men 
Table I Heterogeneity Tests and MR-Egger Intercept of Short Sleep Duration and Frequent Insomnia Symptoms Causally Linked to Ischemic Stroke and Its Subtypes

\begin{tabular}{|l|l|l|l|l|}
\hline Exposure and Outcome & Cochran's $\mathbf{Q}$ & $\boldsymbol{p ~ v a l u e ~}^{\mathbf{a}}$ & Intercept & $\boldsymbol{p}_{\text {value }}^{\mathbf{b}}$ \\
\hline Short sleep duration & & & & \\
$\quad$ Large artery stroke & 26.47 & 0.438 & $-0.01 \mathrm{I}$ & 0.618 \\
Small vessel stroke & 24.19 & 0.508 & 0.015 & 0.480 \\
Cardioembolic stroke & 32.20 & 0.152 & -0.008 & 0.705 \\
Any ischemic stroke & 18.15 & 0.836 & -0.002 & 0.813 \\
\hline Frequent insomnia symptoms & & & & 0.692 \\
Large artery stroke & 76.02 & 0.032 & $-4.74 \times 10^{-3}$ & 0.736 \\
Small vessel stroke & 68.84 & 0.099 & 0.004 & 0.974 \\
Cardioembolic stroke & 52.84 & 0.558 & $-2.60 \times 10^{-4}$ & 0.445 \\
Any ischemic stroke & 74.34 & 0.042 & 0.004 & \\
\hline
\end{tabular}

Notes: ${ }^{a} p$ value for heterogeneity tests; ${ }^{b} p$ value for MR-Egger intercept. Abbreviation: MR, Mendelian randomization.

associated with decreased risk of stroke. ${ }^{5}$ In contrast, a recent systematic review including eight selected studies reported a significant positive association between long sleep duration and the ischemic stroke incidence or ischemic stroke death. ${ }^{1}$ What's more, several epidemiological studies suggested an U-shaped relationship between sleep duration and stroke, indicating that both short and long sleep duration conveyed adverse effects on stroke. ${ }^{6,7,28,29}$ Compared with studies on association of sleep duration with risk of stroke, associations between insomnia or chronotype and risk of stroke have been less investigated. While some observational works have found a higher risk of stroke in insomniacs, ${ }^{1,8,9}$ another study has reported no significant difference of stroke risk in insomniacs compared with noninsomniacs. ${ }^{10}$ However, most of these discordant studies were limited by the susceptibility to bias due to various confounders and not considering specific etiological subtypes of ischemic stroke.

Our MR study provides some evidence for a possible causal relationship of short sleep duration with LAS risk, which is in line with a previous MR study showing greater genetic predisposition to short sleep duration was associated with incident myocardial infarction and coronary artery disease, two etiologically related outcomes for LAS. ${ }^{30}$ Similarly, a previous MR research reported the relevance of frequent insomnia symptoms in prevalent coronary artery disease. ${ }^{16}$ Consistent with our results, a recent MR study using 244 insomnia-associated SNPs, which selected instrumental SNPs in GWAS different from ours, also found a causal association between genetic liability to insomnia and risk of LAS, but not SVS or CES. $^{31}$ The exact mechanisms underlying the associations of short sleep duration and insomnia with LAS risk are unclear. Epidemiological evidence suggests that short sleep duration and insomnia are related to LAS-related risk factors, including hypertension, diabetes, obesity, and dyslipidemia. ${ }^{32-34}$ Furthermore, previous research reported that short sleep duration and insomnia might increase oxidative stress, sympathetic nervous system activity, and systemic inflammation, which could trigger the stroke events. ${ }^{2,31,35}$ Additionally, short sleep duration is also reported being an independent risk factor for atrial fibrillation, ${ }^{36}$ but we did not find a positive causal relationship between short sleep duration and CES, probably due to the relatively small sample size.

One strength of our study is the MR design, which minimizes potential confounding and reverse causality by using genetic instruments for sleep traits. Another strength is the possibility to investigate the associations of sleep phenotypes with ischemic stroke subtypes. One limitation of this study is that SNP effect estimates were largely obtained from the UK Biobank, which might induce selection bias due to low response rates. ${ }^{37}$ Another limitation related to the use of self-reported rather than objective measures to identify genetic variants. However, all of these measures have been validated by accelerometerderived estimates. ${ }^{15-17}$ And the lack of polysomnographic examination of the participants makes it hard to exclude secondary causes and sleep apnea that commonly present in stroke patients. Additionally, generalizability of our analyses is limited because participants were restricted to predominantly of European ancestry to minimize population stratification. Finally, potential pleiotropy might remain. Nonetheless, we conducted a series of sensitivity 
Outcome

Large Artery Stroke

IVW

MR-PRESSO

MR-RAPS

WME

MR-Egger

Small Vessel Stroke

IVW

MR-RAPS

WME

MR-Egger

Cardioembolic Stroke

IVW

MR-RAPS

WME

MR-Egger

\section{Any Ischemic Stroke} IVW

MR-RAPS

WME

MR-Egger
SNPs

OR $(95 \% \mathrm{Cl})$

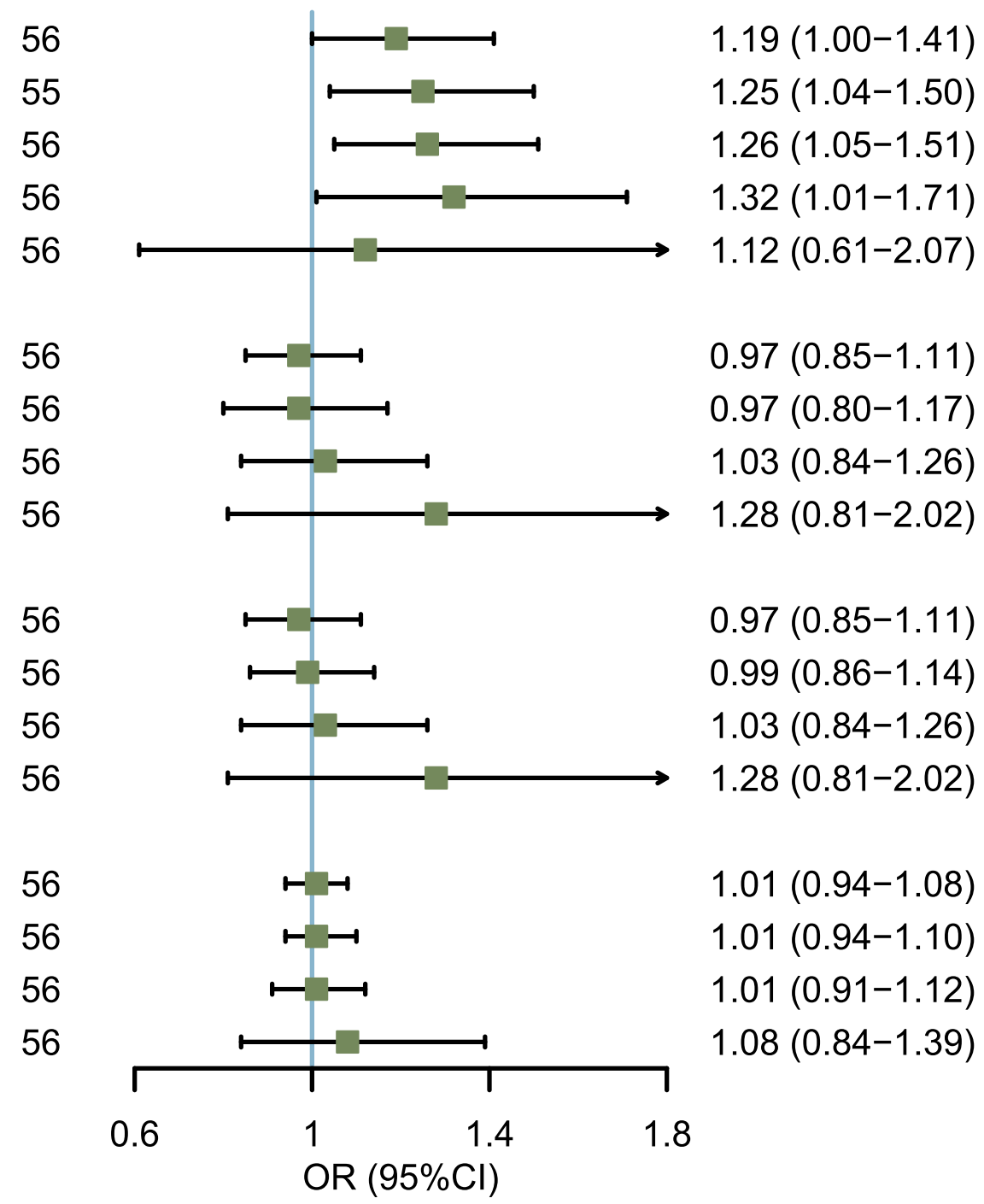

Figure 2 MR estimates of frequent insomnia symptoms with ischemic stroke and its subtypes from the IVW analysis and sensitivity analysis (MR.RAPS, WME, MR-Egger and MR-PRESSO). For MR-PRESSO, outcomes of the outlier-corrected analysis are presented if outliers were detected, otherwise, MR-PRESSO results are not presented. Data is displayed as $\mathrm{OR}$ and $95 \% \mathrm{Cl}$ per doubling of genetic liability for frequent insomnia symptoms.

Abbreviation: MR-PRESSO, MR-Pleiotropy Residual Sum and Outlier.

analyses and outliers detection to explore and account for pleiotropy. The associations of genetic liability to short sleep duration and insomnia symptoms with LAS were overall robust to alternative MR methods and no directional pleiotropy was observed.

\section{Conclusions}

In summary, this study provided suggestive evidence that genetic liability to short sleep duration and insomnia symptoms may be related to a modest increased risk of LAS. Future researches are required to investigate whether improved sleep habits could help to mitigate LAS risk.

\section{Acknowledgments}

We gratefully thank the MEGASTROKE consortium, UK Biobank, HUNT, Partners Biobank, and the 23andMe studies for providing summary statistics data. All MEGASTROKE consortium authors are listed in the online supplementary. The MEGASTROKE project received funding from sources specified at http://www. $\underline{\text { megastroke.org/acknowledgments.html. }}$

\section{Funding}

This study received the support of the Social Welfare Science and Technology Research Project of Zhongshan 
City (2018B1065) and National Natural Science Foundation of China (81501193).

\section{Disclosure}

The authors report no conflicts of interest for this work.

\section{References}

1. Gottlieb E, Landau E, Baxter H, Werden E, Howard ME, Brodtmann A. The bidirectional impact of sleep and circadian rhythm dysfunction in human ischaemic stroke: a systematic review. Sleep Med Rev. 2019;45:54-69. doi:10.1016/j.smrv.2019.03.003

2. Eguchi K, Hoshide S, Ishikawa S, Shimada K, Kario K. Short sleep duration is an independent predictor of stroke events in elderly hypertensive patients. J Am Soc Hypertens. 2010;4(5):255-262. doi:10.1016/j.jash.2010.09.001

3. Ruiter Petrov ME, Letter AJ, Howard VJ, Kleindorfer D. Selfreported sleep duration in relation to incident stroke symptoms: nuances by body mass and race from the REGARDS study. J Stroke Cerebrovasc Dis. 2014;23(2):e123-132. doi:10.1016/j. jstrokecerebrovasdis.2013.09.009

4. Amagai Y, Ishikawa S, Gotoh T, Kayaba K, Nakamura Y, Kajii E. Sleep duration and incidence of cardiovascular events in a Japanese population: the Jichi medical school cohort study. $J$ Epidemiol. 2010;20(2):106-110. doi:10.2188/jea.JE20090053

5. Petrov ME, Howard G, Grandner MA, Kleindorfer D, Molano JR, Howard VJ. Sleep duration and risk of incident stroke by age, sex, and race: the REGARDS study. Neurology. 2018;91(18):e1702e1709. doi:10.1212/WNL.0000000000006424

6. Yin J, Jin X, Shan Z, et al. Relationship of sleep duration with all-cause mortality and cardiovascular events: a systematic review and dose-response meta-analysis of prospective cohort studies. $J \mathrm{Am}$ Heart Assoc. 2017;6(9). doi:10.1161/JAHA.117.005947.

7. Ge B, Guo X. Short and long sleep durations are both associated with increased risk of stroke: a meta-analysis of observational studies. Int J Stroke. 2015;10(2):177-184. doi:10.1111/ijs.12398

8. Wu MP, Lin HJ, Weng SF, Ho CH, Wang JJ, Hsu YW. Insomnia subtypes and the subsequent risks of stroke: report from a nationally representative cohort. Stroke. 2014;45(5):1349-1354. doi:10.1161/ STROKEAHA.113.003675

9. Hsu CY, Chen YT, Chen MH, et al. The association between insomnia and increased future cardiovascular events: a nationwide population-based study. Psychosom Med. 2015;77(7):743-751. doi:10.1097/PSY.0000000000000199

10. Helbig AK, Stockl D, Heier M, Ladwig KH, Meisinger C. Symptoms of insomnia and sleep duration and their association with incident strokes: findings from the population-based MONICA/KORA augsburg cohort study. PLoS One. 2015;10(7):e0134480.

11. Malik R, Chauhan G, Traylor M, et al. Multiancestry genome-wide association study of 520,000 subjects identifies 32 loci associated with stroke and stroke subtypes. Nat Genet. 2018;50(4):524-537. doi:10.1038/s41588-018-0058-3

12. Schulz UG, Rothwell PM. Differences in vascular risk factors between etiological subtypes of ischemic stroke: importance of population-based studies. Stroke. 2003;34(8):2050-2059. doi:10.11 61/01.STR.0000079818.08343.8C

13. Cai H, Cai B, Liu Z, et al. Genetic correlations and causal inferences in ischemic stroke. J Neurol. 2020;267(7):1980-1990. doi:10.1007/ s00415-020-09786-4

14. Bowden J, Del Greco MF, Minelli C, Davey Smith G, Sheehan N, Thompson J. A framework for the investigation of pleiotropy in two-sample summary data Mendelian randomization. Stat Med. 2017;36(11):1783-1802. doi:10.1002/sim.7221
15. Dashti HS, Jones SE, Wood AR, et al. Genome-wide association study identifies genetic loci for self-reported habitual sleep duration supported by accelerometer-derived estimates. Nat Commun. 2019;10 (1):1100. doi:10.1038/s41467-019-08917-4

16. Lane JM, Jones SE, Dashti HS, et al. Biological and clinical insights from genetics of insomnia symptoms. Nat Genet. 2019;51 (3):387-393. doi:10.1038/s41588-019-0361-7

17. Jones SE, Lane JM, Wood AR, et al. Genome-wide association analyses of chronotype in 697,828 individuals provides insights into circadian rhythms. Nat Commun. 2019;10(1):343. doi:10.1038/ s41467-018-08259-7

18. Adams HP Jr, Bendixen BH, Kappelle LJ, et al. Classification of subtype of acute ischemic stroke. Definitions for use in a multicenter clinical trial. TOAST. Trial of org 10172 in acute stroke treatment. Stroke. 1993;24(1):35-41. doi:10.1161/01.STR.24.1.35

19. Krokstad S, Langhammer A, Hveem K, et al. Cohort profile: the HUNT study, Norway. Int J Epidemiol. 2013;42(4):968-977. doi:10.1093/ije/dys095

20. Bowden J, Davey Smith G, Haycock PC, Burgess S. Consistent estimation in mendelian randomization with some invalid instruments using a weighted median estimator. Genet Epidemiol. 2016;40 (4):304-314. doi:10.1002/gepi.21965

21. Bowden J, Davey Smith G, Burgess S. Mendelian randomization with invalid instruments: effect estimation and bias detection through Egger regression. Int J Epidemiol. 2015;44(2):512-525. doi:10.1093/ ije/dyv080

22. Zhao Q, Wang J, Jack Bowden DS. Statistical inference in two-sample summary-data Mendelian randomization using robust adjusted profile score. arXiv. 2018;1801:09652.

23. Verbanck M, Chen CY, Neale B, Do R. Detection of widespread horizontal pleiotropy in causal relationships inferred from Mendelian randomization between complex traits and diseases. Nat Genet. 2018;50(5):693-698. doi:10.1038/s41588-018-0099-7

24. Hemani G, Tilling K, Davey Smith G, Li J. Orienting the causal relationship between imprecisely measured traits using GWAS summary data. PLoS Genet. 2017;13(11):e1007081. doi:10.1371/journal. pgen. 1007081

25. Burgess S, Labrecque JA. Mendelian randomization with a binary exposure variable: interpretation and presentation of causal estimates. Eur J Epidemiol. 2018;33(10):947-952. doi:10.1007/s10654-0180424-6

26. Hemani G, Zheng J, Elsworth B, et al. The MR-base platform supports systematic causal inference across the human phenome. Elife. 2018;7.

27. Shankar A, Syamala S, Kalidindi S, Stadler K. Insufficient rest or sleep and its relation to cardiovascular disease, diabetes and obesity in a national, multiethnic sample. PLoS One. 2010;5(11):e14189. doi:10.1371/journal.pone.0014189

28. Guo Q, Xie W, Peng R, et al. A dose-response relationship between sleep duration and stroke according to nonhealth status in central china: a population-based epidemiology survey. J Stroke Cerebrovasc Dis. 2019;28(7):1841-1852. doi:10.1016/j.jstrokecerebrovasdis.2019.04.016

29. Pergola BL, Moonie S, Pharr J, Bungum T, Anderson JL. Sleep duration associated with cardiovascular conditions among adult Nevadans. Sleep Med. 2017;34:209-216. doi:10.1016/j.sleep.2017.03.006

30. Daghlas I, Dashti HS, Lane J, et al. Sleep duration and myocardial infarction. J Am Coll Cardiol. 2019;74(10):1304-1314. doi:10.1016/ j.jacc.2019.07.022

31. Larsson SC, Markus HS. Genetic liability to insomnia and cardiovascular disease risk. Circulation. 2019;140(9):796-798. doi:10.11 61/CIRCULATIONAHA.119.041830

32. Altman NG, Izci-Balserak B, Schopfer E, et al. Sleep duration versus sleep insufficiency as predictors of cardiometabolic health outcomes. Sleep Med. 2012;13(10):1261-1270. doi:10.1016/j.sleep.2012.08.005

33. Phua CS, Jayaram L, Wijeratne T. Relationship between sleep duration and risk factors for stroke. Front Neurol. 2017;8:392. 
34. Jansen PR, Watanabe K, Stringer S, et al. Genome-wide analysis of insomnia in 1,331,010 individuals identifies new risk loci and functional pathways. Nat Genet. 2019;51(3):394-403. doi:10.1038/ s41588-018-0333-3

35. Zhong X, Hilton HJ, Gates GJ, et al. Increased sympathetic and decreased parasympathetic cardiovascular modulation in normal humans with acute sleep deprivation. J Appl Physiol (1985). 2005;98(6):2024-2032. doi:10.1152/japplphysiol.00620.2004
36. Genuardi MV, Ogilvie RP, Saand AR, et al. Association of short sleep duration and atrial fibrillation. Chest. 2019;156(3):544-552. doi:10.1016/j.chest.2019.01.033

37. Sudlow C, Gallacher J, Allen N, et al. UK biobank: an open access resource for identifying the causes of a wide range of complex diseases of middle and old age. PLoS Med. 2015;12(3):e1001779. doi:10.1371/journal.pmed.1001779

\section{Publish your work in this journal}

Nature and Science of Sleep is an international, peer-reviewed, open access journal covering all aspects of sleep science and sleep medicine, including the neurophysiology and functions of sleep, the genetics of sleep, sleep and society, biological rhythms, dreaming, sleep disorders and therapy, and strategies to optimize healthy sleep.
The manuscript management system is completely online and includes a very quick and fair peer-review system, which is all easy to use. Visit http://www.dovepress.com/testimonials.php to read real quotes from published authors. 